\title{
ANTIMICROBIAL COMPOUNDS FROM RHIZOPHORA STYLOSA
}

\author{
Phan Thi Thanh Huong', Nguyen Van Thanh ${ }^{1, *}$, Chau Ngoc Diep ${ }^{1}$, \\ Nguyen Phuong Thao ${ }^{1}$, Nguyen The Cuong ${ }^{2}$, Nguyen Xuan Cuong ${ }^{1}$, \\ Nguyen Hoai Nam ${ }^{1}$, Chau Van Minh ${ }^{1}$ \\ ${ }^{1}$ Institute of Marine Biochemistry (IMBC), Vietnam Academy of Science and Technology \\ (VAST), 18 Hoang Quoc Viet, Cau Giay, Hanoi, Vietnam \\ ${ }^{2}$ Institute of Ecology and Biological Resources, Vietnam Academy of Science and Technology \\ (VAST), Hanoi, Vietnam. \\ "Email: thanhcmgu@yahoo.com
}

Received: 7 October 2014; Accepted for publication: 25 March 2015

\begin{abstract}
$(6 S, 7 E, 9 R)-6,9$-dihydroxy-4,7-megastigmadien-3-one 9 - $O$ - $[\alpha$-L-arabinopyranosyl-(l $\rightarrow 6)-\beta$ D-glucopyranoside] (1), blumenol A (2), and kaempferol 3-rutinoside (3) were isolated from the methanol extract of the leaves of mangrove plant Rhizophora stylosa Griff. Structural elucidation of the metabolites was carried out by analysis of their spectroscopic data and by comparison with those reported in the literature. All these compounds exhibited antimicrobial activity and were isolated from this genus for the first time.
\end{abstract}

Keywords: Rhizophora stylosa, mangrove, antimicrobial activity, megastigmane, flavonoid.

\section{INTRODUCTION}

Rhizophora stylosa Griff is a common mangrove plant belonging to the family Rhizophoraceae. In Vietnam, this species grows on coasts from Quang Ninh to Ba Ria - Vung Tau provinces [1]. Previous phytochemical and pharmacological studies on this plant reported the isolation of some pentacyclic triterpenoids [2] and flavanol derivatives with antioxidative activity $[3,4]$. Recently, as part of our project directed towards detecting bioactive metabolites from the mangrove plants collected in Xuan Thuy national park, the leaf extract of $R$. stylosa showed cytotoxic and antimicrobial activities. We wish to report herein the antimicrobial compounds isolated from the extract of $R$. stylosa leaves.

\section{EXPERIMENTAL}

\subsection{General experimental procedures}

NMR spectra were recorded on a Bruker AM500 FT-NMR spectrometer (Bruker, Billerica, MA, U.S.A.) using TMS as an internal standard. Column chromatography (CC) was performed 
using a silica gel (Kieselgel 60, 70-230 mesh and 230-400 mesh, Merck, Darmstadt, Germany) or YMC RP-18 resins (30 - $50 \mu \mathrm{m}$, Fuji Silysia Chemical Ltd, Aichi, Japan). Thin layer chromatography (TLC) used pre-coated silica gel $60 \mathrm{~F}_{254}$ (1.05554.0001, Merck, Darmstadt, Germany) and RP-18 $\mathrm{F}_{254 \mathrm{~s}}$ plates (1.15685.0001, Merck, Darmstadt, Germany) and compounds were visualized by spraying with aqueous $10 \% \mathrm{H}_{2} \mathrm{SO}_{4}$ and heating for 3-5 minutes.

\subsection{Plant material}

The samples of the mangrove plant Rhizophora stylosa were collected in July 2013 at Xuan Thuy national park, Nam Dinh province, Vietnam and identified by Dr. Nguyen The Cuong. A voucher specimen (no. XT_CB05C) was maitained at the IMBC, VAST.

\subsection{Extraction and isolation}

Dried leaves of $R$. stylosa $(1.8 \mathrm{~kg})$ were powdered and extracted with hot $\mathrm{MeOH}$ (three times at $50^{\circ} \mathrm{C}$ for $6 \mathrm{~h}$ each) to give a $\mathrm{MeOH}$ residue $(200 \mathrm{~g}, \mathrm{~A})$ after removal of the solvent in a vacuum. This extract was suspended in water and partitioned in turn with $n$-hexane and $\mathrm{CH}_{2} \mathrm{Cl}_{2}$ to provide the corresponding extracts: $n$-hexane $(\mathrm{H}, 80 \mathrm{~g}), \mathrm{CH}_{2} \mathrm{Cl}_{2}(\mathrm{C}, 20 \mathrm{~g})$, and a water layer. Extracts $\mathrm{H}$ and $\mathrm{C}$ were combined and crudely separated by silica gel $\mathrm{CC}$ using a gradient concentration of $\mathrm{MeOH}$ in $\mathrm{CH}_{2} \mathrm{Cl}_{2}(0-100 \%)$ to obtain seven fractions (H1-H7). Fraction $\mathrm{H} 4$ $(4.1 \mathrm{~g})$ was further separated by YMC RP-18 CC and eluted with $\mathrm{MeOH}-$ water $(2.5: 1, \mathrm{v} / \mathrm{v})$, followed by silica gel CC with $n$-hexane-acetone (3:1, v/v) to give compound $2(5.0 \mathrm{mg})$.

The water layer was passed through a Diaion HP-20 column using stepwise elution with water-MeOH (10:0, 2.5:7.5, 5:5, 7.5:2.5, and 0:10, v/v) to obtain four fractions (W1-W4) after removal of the fraction eluted with only water. Fraction W2 $(1.5 \mathrm{~g})$ was purified by silica gel CC and eluted with $\mathrm{CH}_{2} \mathrm{Cl}_{2}-\mathrm{MeOH}-$ water $(5: 1: 0.1, \mathrm{v} / \mathrm{v})$, followed by YMC RP-18 CC and elution with $\mathrm{MeOH}$-water (1:2, v/v) to provide compounds $\mathbf{1}(7.0 \mathrm{mg})$ and $\mathbf{3}(30 \mathrm{mg})$.

$(6 S, 7 E, 9 R)-6,9-d i h y d r o x y-4,7-m e g a s t i g m a d i e n-3-o n e ~ 9-O-[\alpha$-L-arabinopyranosyl-( $1 \rightarrow 6)-\beta$ D-glucopyranoside] (1): Amophous powder; ${ }^{1} \mathrm{H}-\mathrm{NMR}$ (500 MHz, $\left.\mathrm{CD}_{3} \mathrm{OD}\right)$ and ${ }^{13} \mathrm{C}-\mathrm{NMR}(125$ $\mathrm{MHz}, \mathrm{CD}_{3} \mathrm{OD}$ ) see table 1.

Blumenol A (2): Amophous powder; ${ }^{1} \mathrm{H}-\mathrm{NMR}$ (500 MHz, $\left.\mathrm{CD}_{3} \mathrm{OD}\right)$ and ${ }^{13} \mathrm{C}-\mathrm{NMR}(125$ $\mathrm{MHz}, \mathrm{CD}_{3} \mathrm{OD}$ ) see table 1.

Kaempferol 3-rutinoside (3): Yellow amorphous powder; ${ }^{1} \mathrm{H}-\mathrm{NMR}\left(500 \mathrm{MHz}, \mathrm{CD}_{3} \mathrm{OD}\right)$ and ${ }^{13} \mathrm{C}$-NMR $\left(125 \mathrm{MHz}, \mathrm{CD}_{3} \mathrm{OD}\right)$ see table 1 .

\subsection{Antimicrobial activity test}

Antimicrobial activity test was carried out at the Department of Experimental Biology, Institute of Natural Products Chemistry, VAST, using the method described by Vanden Berghe, Vlietinck, and McKane, Kandel [5,6]. This experiment was performed by microdilution technique on 96-well microtiter plate. Two gram-negative bacteria (Escherichia coli, Pseudomonas aeruginosa), two gram-positive bacteria (Bacillus subtillis, Staphylococcus aureus subsp. aureus) and four fungal strains (Aspergillus niger, Fusarium oxysporum, Candida albicans, Saccharomyces cerevisiae) were employed to determine antimicrobial activity and minimum inhibitory concentration (MIC). The reference antibiotics were streptomycin (4 mM), tetracyclin $(10 \mathrm{mM})$ and nystatin $(4 \mathrm{mM})$. Fungi and bacteria were cultured in nutrient media. 
The test microorganisms were activated before the testing in fluid nutrient media. MIC is defined as the lowest concentration of antibiotic completely inhibiting visible growth of bacteria.

\section{RESULT AND DISCUSSION}<smiles>[R]O[C@@H](C)/C=C/[C@@]1(O)[C@@H](C)C=C([R2])C[C@]1([2H])[3H]</smiles>

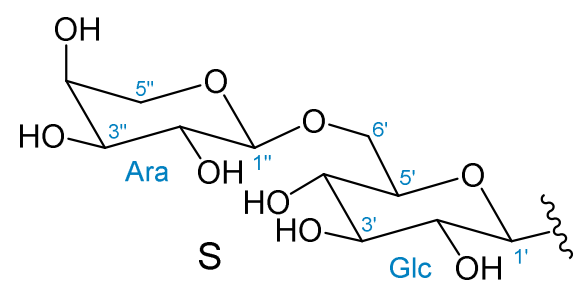

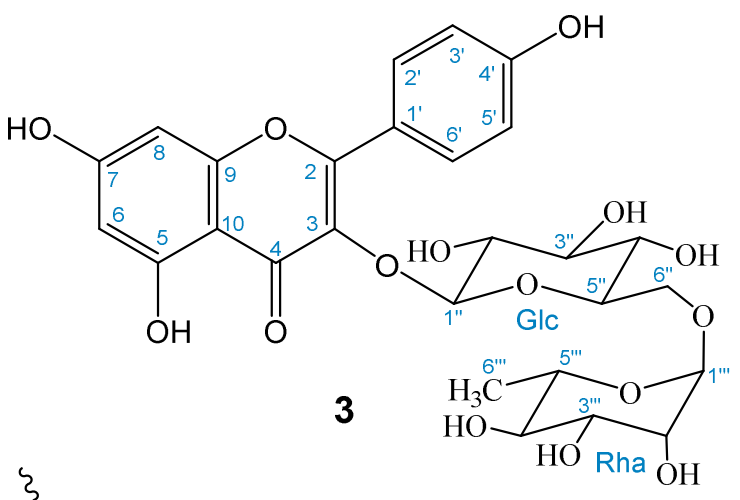

Figure 1. Structrure of compounds 1 - 3.

Compound 1 was obtained as an amorphous powder. The ${ }^{1} \mathrm{H}-\mathrm{NMR},{ }^{13} \mathrm{C}$ NMR, and DEPT spectra indicated that $\mathbf{1}$ was a megastigmane diglycoside. The ${ }^{13} \mathrm{C}$ NMR spectrum (Table 1), in combination with the HSQC and DEPT spectra exhibited 11 signals assignable to two sugar moieties. The remaining 13 signals being assigned to a megastigmane skeleton, including one carbonyl carbon at $\delta 201.28$, four olefinic carbons at $\delta 127.24,167.24,131.67,134.96$, one oxygenated quaternary carbon at $\delta 80.09$, one oximethine carbon at $\delta 76.76$, one methylen carbon at $\delta 50.77$, one quaternary carbon at $\delta 42.48$, and four methyl carbon at $\delta 19.97,21.07$, 23.46, 24.70. The ${ }^{1} \mathrm{H}$ NMR spectrum displayed signals for two tertiary methyl groups at $\delta 1.05$ $(3 \mathrm{H}, \mathrm{s}), 1.06(3 \mathrm{H}, \mathrm{s})$, a secondary methyl at $\delta 1.31(3 \mathrm{H}, \mathrm{d}, J=6.0 \mathrm{~Hz})$, a methyl attached to an olefinic carbon at $\delta 1.94(3 \mathrm{H}, \mathrm{d}, J=1.5 \mathrm{~Hz})$, a pair of isolated methylene protons centered at $\delta$ $2.18(1 \mathrm{H}, \mathrm{d}, J=17.0 \mathrm{~Hz}), 2.53(1 \mathrm{H}, \mathrm{d}, J=17.0 \mathrm{~Hz})$, an oximethine proton at $\delta 4.46(1 \mathrm{H}, \mathrm{m})$ and three olefinic protons at $\delta 5.91(1 \mathrm{H}, \mathrm{t}, J=1.5 \mathrm{~Hz}), 5.86(1 \mathrm{H}$, overlap), $5.87(1 \mathrm{H}$, overlap). In the HMBC spectrum, long-range correlations were observed between the following protons and carbons: H-2 and C-1, C-3, C-4, C-6, C-11; H-4 and C-2, C-6, C-13; H-7 and C-5, C-6, C-8, C9; H-8 and C-6, C-7, C-9; H-10 and C-8, C-9; H-11, H-12 and C-1, C-2, C-6; H-13 and C-4, C$5, \mathrm{C}-6$, indicated that the aglycone structure of $\mathbf{1}$ is 6,9-dihydroxy-4,7-megastigmadien-3-one. The ${ }^{1} \mathrm{H}$ NMR also showed an anomeric proton signal at $\delta 4.37(1 \mathrm{H}, \mathrm{d}, J=8.0 \mathrm{~Hz})$ and two oximethylene protons at $\delta 3.71(1 \mathrm{H}, \mathrm{dd}, J=5.3 ; 11.0 \mathrm{~Hz}), 4.08(1 \mathrm{H}, \mathrm{dd}, J=2.0 ; 11.0 \mathrm{~Hz})$ were indicative to the presence of $\beta$-D-glucopyranosyl moiety. The second anomeric proton was observed at $\delta 4.30(1 \mathrm{H}, \mathrm{d}, J=6.5 \mathrm{~Hz})$, together with two oxymethylene protons observed at $\delta$ $3.54,3.88(1 \mathrm{H}, \mathrm{dd}, J=3.0 ; 12.5 \mathrm{~Hz})$ which was characteristic for $\alpha$-L-arabinopyranosyl moiety. The glycosidic positions were established by the HMBC experiment, in which the long-range correlations were observed between the H-1' $(\delta 4.37)$ of D-glucose and the C-9 $(\delta 76.76)$ of the aglycon, and between the H-1"' $(\delta 4.30)$ of L-arabinose and the C-6' $(\delta$ 69.55) of D-glucose. Therefore, 1 was elucidated as $(6 S, 7 E, 9 R)-6,9$-dihydroxy-4,7-megastiymadien-3-one 9- $O$-[ $\alpha$-Larabinopyranosyl-(l $\rightarrow 6)-\beta$-D-glucopyranoside] [7], which is isolated for the first time from Rhizophora genus. 
Table 1. The NMR data of compounds $\mathbf{1 ,} \mathbf{2}$, and $\mathbf{3}$.

\begin{tabular}{|c|c|c|c|c|c|c|c|}
\hline \multirow{2}{*}{ No } & \multicolumn{2}{|r|}{1} & \multicolumn{2}{|r|}{2} & \multirow{2}{*}{ No } & \multicolumn{2}{|r|}{3} \\
\hline & $\delta_{C}{ }^{a, b}$ & $\delta_{\mathrm{H}}^{\mathrm{a}, \mathrm{c}}$ & $\boldsymbol{\delta}_{\mathrm{C}}^{\mathrm{a}, \mathrm{b}}$ & $\delta_{\mathrm{H}}^{\mathrm{a}, \mathrm{c}}$ & & $\boldsymbol{\delta}_{\mathrm{C}}^{\mathrm{a}, \mathrm{b}}$ & $\delta_{\mathrm{H}}^{\mathrm{a}, \mathrm{c}}$ \\
\hline 1 & 42.48 & 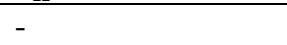 & 42.42 & 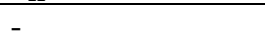 & 2 & 159.06 & - \\
\hline 2 & 50.77 & $\begin{array}{l}2.18(\mathrm{~d}, 17.0) \\
2.53(\mathrm{~d}, 17.0)\end{array}$ & 50.73 & $\begin{array}{l}2.18(\mathrm{~d}, 17.0) \\
2.53(\mathrm{~d}, 17.0)\end{array}$ & 3 & 135.45 & - \\
\hline 3 & 201.28 & - & 201.27 & - & 4 & 179.07 & - \\
\hline 4 & 127.24 & $5.91(\mathrm{t}, 1.5)$ & 127.10 & $5.90(\mathrm{t}, 1.5)$ & 5 & 162.83 & - \\
\hline 5 & 167.24 & - & 167.48 & - & 6 & 100.87 & $6.18(\mathrm{~d}, 2.0)$ \\
\hline 6 & 80.09 & - & 79.95 & - & 7 & 168.75 & - \\
\hline 7 & 131.67 & $5.86^{\mathrm{d}}$ & 130.10 & $5.81^{\mathrm{d}}$ & 8 & 95.55 & $6.36(\mathrm{~d}, 2.0)$ \\
\hline 8 & 134.96 & $5.87^{\mathrm{d}}$ & 136.92 & $5.82^{\mathrm{d}}$ & 9 & 158.75 & - \\
\hline 9 & 76.76 & $4.46(\mathrm{~m})$ & 68.72 & $4.34(\mathrm{dd}, 4.5 ; 6.5)$ & 10 & 104.91 & - \\
\hline 10 & 21.07 & $1.31 \mathrm{~d}, 6.0)$ & 23.82 & $1.26(\mathrm{~d}, 6.5)$ & $1^{\prime}$ & 122.79 & - \\
\hline 11 & 23.46 & $1.06(\mathrm{~s})$ & 23.46 & $1.06(\mathrm{~s})$ & $2^{\prime}$ & 132.32 & $8.07(\mathrm{~d}, 9.0)$ \\
\hline 12 & 24.70 & $1.05(\mathrm{~s})$ & 24.47 & $1.03(\mathrm{~s})$ & $3^{\prime}$ & 116.17 & $6.90(\mathrm{~d}, 9.0)$ \\
\hline 13 & 19.97 & $1.94(\mathrm{~d}, 1.5)$ & 19.56 & $1.94(\mathrm{~d}, 1.5)$ & $4^{\prime}$ & 161.55 & - \\
\hline 1 ' & 102.59 & $4.37(\mathrm{~d}, 8.0)$ & & & $5^{\prime}$ & 116.17 & $6.90(\mathrm{~d}, 9.0)$ \\
\hline $2^{\prime}$ & 75.20 & $3.20(\mathrm{~m})$ & & & $6^{\prime}$ & 132.32 & $8.07(\mathrm{~d}, 9.0)$ \\
\hline $3^{\prime}$ & 77.94 & $3.38^{\mathrm{d}}$ & & & $1^{\prime \prime}$ & 104.91 & $5.10(\mathrm{~d}, 7.5)$ \\
\hline $4^{\prime}$ & 71.57 & $3.37^{\mathrm{d}}$ & & & $2^{\prime \prime}$ & 75.77 & $3.47^{\mathrm{d}}$ \\
\hline 5 & 76.79 & $3.41(\mathrm{~m})$ & & & $3^{\prime \prime}$ & 78.21 & $3.44^{\mathrm{d}}$ \\
\hline $6^{\prime}$ & 69.55 & $\begin{array}{l}3.71(\mathrm{dd}, 5.3 ; 11.0) \\
4.08(\mathrm{dd}, 2.0 ; 11.0)\end{array}$ & & & $4^{\prime \prime}$ & 71.44 & $3.28^{\mathrm{d}}$ \\
\hline $1 "$ & 105.22 & $4.30(\mathrm{~d}, 6.5)$ & & & $5^{\prime \prime}$ & 77.22 & $3.36^{\mathrm{d}}$ \\
\hline $2^{\prime \prime}$ & 74.17 & $3.55^{\mathrm{d}}$ & & & $6^{\prime \prime}$ & 68.61 & $\begin{array}{l}3.40^{\mathrm{d}} \\
3.83(\mathrm{dd} 11.0,0.5)\end{array}$ \\
\hline $3 "$ & 72.34 & $3.62(\mathrm{~m})$ & & & $1^{\prime \prime \prime}$ & 102.44 & $4.54(\mathrm{~d}, 1.5)$ \\
\hline $4 "$ & 69.49 & $3.82(\mathrm{~m})$ & & & $2^{\prime \prime \prime}$ & 72.09 & $3.66(\mathrm{dd}, 1.5,3.5)$ \\
\hline $5 "$ & 66.74 & $\begin{array}{l}3.54^{\mathrm{d}} \\
3.88(\mathrm{dd}, 3.0 ; 12.5)\end{array}$ & & & $3^{\prime \prime \prime}$ & 72.31 & $3.55(\mathrm{dd}, 3.5,9.5)$ \\
\hline & & & & & $4^{\prime \prime \prime}$ & 73.92 & $3.32^{\mathrm{d}}$ \\
\hline & & & & & $5^{\prime \prime \prime}$ & 69.73 & $3.48^{\mathrm{d}}$ \\
\hline & & & & & $6^{\prime \prime \prime}$ & 17.91 & $1.15(\mathrm{~d}, 6.5)$ \\
\hline
\end{tabular}

${ }^{a}$ recorded in $\mathrm{CD}_{3} \mathrm{OD},{ }^{b} 125 \mathrm{MHz},{ }^{c} 500 \mathrm{MHz},{ }^{d}$ overlapped signal

Compound 2 was isolated as an amorphous powder. Its NMR data are very similar to those of 1, except for the absence of signals for a diglycoside moiety at C-9 position. The upfield-shift of the $\mathrm{H}-9$ signal at $\delta 4.34(1 \mathrm{H}, \mathrm{dd}, J=4.5 ; 6.5 \mathrm{~Hz})$ and of the C-9 signal at $\delta 68.72$ indicated that a hydroxyl group was attached at the C-9 position. Thus, compound $\mathbf{2}$ was identified as blumenol A by comparison of its NMR data with the reported data [8].

Compound $\mathbf{3}$ was obtained as a yellow amorphous powder. It was identified as a C-3 diglycosylated kaempferol, as revealed by the ${ }^{1} \mathrm{H}-\mathrm{NMR}$ (see table 1) signals of an AX spin system at $\delta 6.18(1 \mathrm{H}, \mathrm{d}, \mathrm{J}=2.0 \mathrm{~Hz}, \mathrm{H}-6), 6.36(1 \mathrm{H}, \mathrm{d}, J=2.0 \mathrm{~Hz}, \mathrm{H}-8)$, an AA'BB' spin system at $\delta 8.07\left(2 \mathrm{H}, \mathrm{d}, \mathrm{J}=9.0 \mathrm{~Hz}, \mathrm{H}-2^{\prime}, \mathrm{H}-6^{\prime}\right), 6.90\left(1 \mathrm{H}, \mathrm{d}, J=9.0 \mathrm{~Hz}, \mathrm{H}-3^{\prime}, \mathrm{H}-5^{\prime}\right)$. The ${ }^{1} \mathrm{H}-\mathrm{NMR}$ spectrum also supported the presence of rutinoside moiety with a glucose anomeric proton signal at $\delta 5.10(1 \mathrm{H}, \mathrm{d}, J=7.5 \mathrm{~Hz})$, a rhamnose $\mathrm{H}-1{ }^{\prime \prime}$, signal at $\delta 4.54(1 \mathrm{H}, \mathrm{d}, J=1.5 \mathrm{~Hz})$ and a doublet signal of methyl group of rhamnose at $\delta 1.15(3 \mathrm{H}, \mathrm{d}, J=6.5 \mathrm{~Hz})$. From the coupling constants of the anomeric protons, the configuration at C-1" glucose and C-1" " rhamnose were 
determined to be the $\beta$ - and $\alpha$-configuration, respectively. In the HMBC spectrum, a cross-peak between $\mathrm{H}-1$ " $(\delta 5.10)$ and C-3 $(\delta 135.45)$ confirmed that the glucosyl moiety was linked at C-3 of the flavonol skeleton. In addition, a long range correlation between H-1 "' $(\delta 4.54)$ and C-6", ( $\delta$ 68.61) indicated that rhamnose unit was attached at C-6" of glucose. Accordingly, the structure of $\mathbf{3}$ was established as kaempferol 3-rutinoside [9].

Table 2. Antimicrobial activity of compounds $\mathbf{1}-\mathbf{3}$.

\begin{tabular}{|c|c|c|c|c|c|c|c|c|}
\hline \multirow{3}{*}{ Compounds } & \multicolumn{7}{|c|}{ MIC $(\mu \mathbf{g} / \mathbf{m l})$} \\
\cline { 2 - 9 } & \multicolumn{2}{|c|}{$\mathbf{G r}(-)$ bacteria } & \multicolumn{2}{|c|}{ Gr(+) bacteria } & \multicolumn{2}{|c|}{ Mold } & \multicolumn{2}{|c|}{ Yeast } \\
\cline { 2 - 9 } & $\begin{array}{c}E \\
\text { coli }\end{array}$ & $\begin{array}{c}P . \\
\text { aeruginosa }\end{array}$ & $\begin{array}{c}\text { B. } \\
\text { subtillis }\end{array}$ & $\begin{array}{c}S . \\
\text { aureus }\end{array}$ & $\begin{array}{c}A . \\
\text { niger }\end{array}$ & $\begin{array}{c}F \\
\text { oxysporum }\end{array}$ & $\begin{array}{c}\text { S. } \\
\text { cerevisiae }\end{array}$ & $\begin{array}{c}\text { C. } \\
\text { albicans }\end{array}$ \\
\hline $\mathbf{1}$ & $(-)$ & $(-)$ & $(-)$ & $(-)$ & $\mathbf{1 0 0}$ & $\mathbf{5 0}$ & $(-)$ & $(-)$ \\
\hline $\mathbf{2}$ & $(-)$ & $(-)$ & $(-)$ & $(-)$ & $\mathbf{1 0 0}$ & $\mathbf{5 0}$ & $(-)$ & $(-)$ \\
\hline $\mathbf{3}$ & $(-)$ & $(-)$ & $(-)$ & $(-)$ & $(-)$ & $\mathbf{5 0}$ & $(-)$ & $(-)$ \\
\hline
\end{tabular}

Table 2 shows the MIC results for compounds $\mathbf{1}-\mathbf{3}$ against eight microorganisms. The antimicrobial studies showed that all the compounds were active against $F$. oxysporum strains with MIC of $50 \mu \mathrm{g} / \mathrm{ml}$ and compounds 1,2 inhibited the growth of A. niger with MIC of 100 $\mu \mathrm{g} / \mathrm{ml}$. The remaining microorganisms were not susceptible to these compounds.

\section{CONCLUSIONS}

Phytochemical study on the methanol extract of the leaves of mangrove plant Rhizophora stylosa led to the isolation of three antimicrobial compounds, $(6 S, 7 E, 9 R)$-6,9-dihydroxy-4,7megastigmadien-3-one 9- $O$-[ $\alpha$-L-arabinopyranosyl- $(1 \rightarrow 6)-\beta$-D-glucopyranoside $](\mathbf{1})$, blumenol A (2), and kaempferol 3-rutinoside (3). Their structures were identified by comparison of spectroscopic data with those reported in the literature. This is the first report for the isolation of these compounds from this species.

Acknowledgements. This work was financially supported by Vietnam Academy of Science and Technology (Project code: VAST.ĐTCB 02/13-14). The authors are grateful to Institute of Chemistry, VAST for measuring NMR data as well as Institute of Natural Products Chemistry, VAST for testing antimicrobial activity.

\section{REFERENCES}

1. Pham Hoang Ho - Vietnamese plants 2, Young pblisher, Ho Chi Minh city, 2003, p. 112.

2. Dong-Li Li, Xiao-Ming Li, Bin-Gui Wang - Pentacyclic triterpenoids from the mangrove plant Rhizophora stylosa, Natural Product Research 22 (2008) 808-813.

3. Kensaku Takara, Ayako Kuniyoshi, Koji Wada, Kazuhiko Kinjyo, Hironori Iwasaki Antioxidative Flavan-3-ol Glycosides from Stems of Rhizophora stylosa, Biosci Biotechnol Biochem. 72 (2008) 2191-2194. 
4. Dong-Li Li, Xiao-Ming Li, Ze-Yu Peng and Bin-Gui Wang - Flavanol Derivatives from Rhizophora stylosa and Their DPPH Radical Scavenging Activity, Molecules 12 (2007) 1163-1169.

5. Vanden Berghe D. A., Vlietinck A. J. - Screening methods for antibacterial and antiviral agents from higher plants 6, Academic Press, San Diego, 1991, p. 47-49.

6. Mckane L. And Kandel J. - Microbiology: essentials and applications, McGraw-Hill, New York, 1996, p. 335.

7. Noriko Matsuda, Kyouko Isawa, Masao Kikuchi - Megastigmane glycosides from Lonicera gracilipes var. Glandulosa, Phytochemistry 45 (1997) 777-779.

8. Antonio G. González, José A. Guillermo, Angel G. Ravelo, Ignacio A. Jimenez, Mahabir P. Gupta - 4,5-Dihydroblumenol A, a new nor-isoprenoid from Perrottetia multiflora, J. Nat. Prod. 57 (1994) 400-402.

9. Kohei Kazuma, Naonobu Noda, Masahiko Suzuki - Malonylated flavonol glycosides from the petals of Clitoria ternatea, Phytochemistry 62 (2003) 229-237.

\section{TÓM TẮT}

\section{CÁC HỢP CHẤT CÓ HOẠT TÍNH KHÁNG VI SINH VẠT KIỂM ĐỊNH PHÂN LẬP ĐƯợC TƯ CÂY ĐƯỚC VÒI - RHIZOPHORA STYLOSA}

Phan Thị Thanh Hương ${ }^{1}$, Nguyễn Văn Thanh ${ }^{1,{ }^{*}}$, Châu Ngọc Điệp ${ }^{1}$, Nguyễn Phương Thảo ${ }^{1}$, Nguyễn Thế Cường ${ }^{2}$, Nguyễn Xuân Cường ${ }^{1}$, Nguyễn Hoài Nam ${ }^{1}$, Châu Văn Minh ${ }^{1}$

${ }^{I}$ Viện Hóa sinh biển (IMBC), Viện HLKHCNVN, 18 Hoàng Quốc Việt, Cầu Giấy, Hà Nội

${ }^{2}$ Viện Sinh thái và Tài nguyên Sinh vật, Viện HLKHCNVN

"Email: thanhcmgu@yahoo.com

Bằng các phương pháp sắc ký kết hợp, ba hợp chất $(6 S, 7 E, 9 R)-6,9$-dihydroxy-4,7megastigmadien-3-one 9- $O$-[ $\alpha$-L-arabinopyranosyl-(l $\rightarrow 6)-\beta$-D-glucopyranoside] $(\mathbf{1})$, blumenol $\mathrm{A}(\mathbf{2})$ và kaempferol 3-rutinoside $(3)$ được phân lập từ cặn chiết metanol của lá cây Đước vòi Rhizophora stylosa Griff. Cấu trúc hóa học của các hợp chất 1-3 được xác định bằng các phương pháp phổ cộng hưởng từ hạt nhân (NMR) kết hợp so sánh với các số liệu đã được công bố. Tất cả ba hợp chất đều thể hiện hoạt tính kháng vi sinh vật kiểm định và đây là lần đầu tiên các hợp chất này được phân lập từ chi Rhizophora.

Tù khóa: Rhizophora stylosa, cây ngập mặn, kháng vi sinh vật kiểm định, megastigmane, flavonoid. 